\title{
eJRIEPS
}

Ejournal de la recherche sur l'intervention en éducation physique et sport

38 | 2016

Varia

\section{Postures et gestes professionnels de formateurs dans l'accompagnement professionnel d'enseignants du premier degré}

Anne Jorro

\section{(2) OpenEdition}

1 Journals

\section{Édition électronique}

URL : https://journals.openedition.org/ejrieps/906

DOI : 10.4000/ejrieps.906

ISSN : 2105-0821

Éditeur

ELLIADD

Référence électronique

Anne Jorro, «Postures et gestes professionnels de formateurs dans l'accompagnement professionnel d'enseignants du premier degré », eJRIEPS [En ligne], 38 | 2016, mis en ligne le 01 avril 2016, consulté le 21 septembre 2021. URL : http://journals.openedition.org/ejrieps/906 ; DOI : https://doi.org/ 10.4000/ejrieps.906

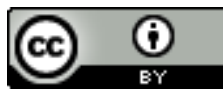

La revue eJRIEPS est mise à disposition selon les termes de la Creative Commons Attribution 4.0 International License. 
eJRIEPS 38 avril 2016

Postures et gestes professionnels de formateurs dans l'accompagnement

professionnel d'enseignants du premier degré

Anne Jorro

Cnam, CRF(centre de recherche sur la formation), Paris, France

\section{Résumé}

La professionnalité du formateur accompagnant les débuts dans le métier d'enseignants du premier degré est étudiée du point de vue des postures professionnelles mobilisées. Comme "expression primordiale de l'agir» (Merleau-Ponty, 1960), la posture professionnelle engloberait des gestes professionnels qui façonnent les interactions plus ou moins propices, plus ou moins opportunes, plus ou moins efficaces en situation de formation. A la suite des travaux issus de l'ethnologie, de la phénoménologie et de l'anthropologie historique allemande, cet article propose une analyse à grains fins de postures et gestes professionnels de formateurs dans des situations d'accompagnement professionnel. Le présent texte prend appui sur une recherche collaborative conduite avec 13 formateurs du premier degré autour de 10 situations d'analyse de pratiques.

Mots clés : professionnalité, formateur, postures professionnelles, gestes professionnels, accompagnement, enseignant débutant.

La professionnalisation des acteurs de la formation a été particulièrement questionnée du point de vue des compétences et des identités professionnelles depuis une bonne vingtaine d'années. Dans cet article, nous voudrions situer le questionnement sur l'agir professionnel du formateur dans l'accompagnement d'enseignants du premier degré, en étudiant son pouvoir d'agir et, en particulier, la place de la corporéité dans l'activité du formateur.

L'agir professionnel d'un formateur accompagnant les débuts dans le métier d'enseignants du premier degré n'est pas réductible au fait de tenir un rôle, à celui de superviser des activités ou en encore de prescrire des règles, mais bien plus à celui d'accompagner un pair en ayant soin de favoriser une relation formative dans laquelle la posture du formateur joue un rôle important. En tant que «système d'attitudes et de regards vis-à-vis des partenaires, des situations, des objets » dans une situation donnée (Ardoino, 1990), la 


\section{eJRIEPS 38 avril 2016}

posture permet de souligner l'intention de l'acteur vis-vis d'autrui au moment où il agit. Comme « expression primordiale de l'agir » (Merleau-Ponty, 1960), la posture engloberait des gestes professionnels qui façonnent les interactions plus ou moins propices, plus ou moins opportunes, plus ou moins efficaces en situation de formation. Autrement dit, les postures et les gestes professionnels des formateurs constitueraient dans le cadre de cette recherche, des clés de compréhension d'une activité professionnelle située. A la suite des travaux issus de l'ethnologie, de la phénoménologie et de l'anthropologie historique allemande, cet article propose une analyse à grains fins de postures et gestes professionnels de formateurs dans des situations d'accompagnement professionnel. Le présent texte prend appui sur une recherche collaborative conduite avec 13 formateurs du premier degré autour de 10 situations d'analyse de pratiques.

\section{Place du corps dans l'agir social}

Le fait de concevoir la place du corps dans l'agir social permet de questionner le pouvoir d'agir d'un acteur. Ce pouvoir d'agir comprend des dimensions cognitives et sociales indissociables puisque dans l'interaction entre l'acteur et le milieu, l'activité est ajustée selon la perception de ces interactions par l'acteur. Autrement dit, penser le pouvoir d'agir du formateur du point de vue de la corporéité implique une lecture plurielle de l'activité dans ses fondements cognitifs, kinésiques, sociaux, affectifs, langagiers,...

La corporéité de l'activité du formateur reste encore peu interrogée par la recherche. Un certain nombre de travaux portant sur l'activité enseignante ont été développés dans plusieurs champs disciplinaires soit pour contribuer à la caractérisation d'une matrice de l'agir professoral, soit pour identifier un geste précis. Ainsi, en didactique du français Bucheton (2009) modélise l'agir enseignant avec les gestes de tissage, d'étayage, d'atmosphère et de pilotage, Jorro (2004) propose une matrice de l'agir professionnel de l'enseignant avec les gestes langagiers, les gestes de mise en scène des savoirs, les gestes d'ajustement de l'activité et les gestes éthiques. En didactique des mathématiques, Sensevy (2001) caractérise quatre gestes d'enseignement tels définir, dévoluer, évaluer, institutionnaliser ; en EPS Brière-Guenoun (2015) met en évidence la part des dilemmes du professeur dans les gestes didactiques de métier. D'autres recherches soulignent les effets des gestes langagiers de l'enseignant dans la régulation des apprentissages (Mercier-Brunel \& Jorro, 2009 et 2011) et décrivent un geste professionnel particulier comme le geste d'écrire au tableau (Hassan, 2010) ou le geste du maquignon en EPS (Loquet, 2006). Depuis les travaux précurseurs de Pujade -Renaud, (1981) sur la place du 


\section{eJRIEPS 38 avril 2016}

corps de l'enseignant en classe, en particulier sur la puissance corporelle de l'enseignant dans sa stature verticale et dans sa force de frappe verbale, les travaux qui se sont développés depuis une dizaine d'années soulignent la particularité performative de certains gestes auprès des élèves, du fait qu'ils peuvent induire un comportement particulier chez ces derniers.

Du côté du champ de la formation des enseignants, les recherches sont peu nombreuses. Alin (2010) distingue les actions génériques qui sont mobilisées au quotidien comme le fait de parler, écrire, montrer, écouter, regarder... avec les micro gestes techniques de l'enseignant comme "faire faire, ordonner, expliquer, démontrer, manipuler.. » qui représenteraient l'expertise du métier d'enseignant. D'autres recherches (Jorro, 2002 ; Jorro \& Pana-Martin, 2009 ; Pana-Martin, 2015) tendent vers la caractérisation de l'agir professionnel du formateur accompagnant des enseignants débutants. Le formateur est alors considéré comme un acteur social engagé dans un champ de pratiques formatives. II agit en fonction des expériences réfléchies comme étant déterminantes pour les enseignants et dans la perspective de leur développement professionnel. Son agir professionnel se spécifie par les finalités propres du métier de formateur. C'est donc un agir singulier qui puise dans un genre professionnel qui n'est pas stabilisé (faute d'une reconnaissance des institutions) mais qui tend à apparaître avec l'émergence de plusieurs secteurs d'activités, en particulier le champ de la formation d'adultes, le champ de la santé où existent les référentiels de compétences du formateur, le titre professionnel de formateur d'adultes...

L'analyse de l'agir professionnel offre donc la possibilité de comprendre ce qui le spécifie, de porter attention aux invariants de l'activité du formateur et de questionner les modalités d'appropriation du genre à travers le style professionnel (Clot, 1999). Bien que ce genre reste clandestin dans le champ de la formation des formateurs d'enseignants, il correspond à la définition donnée par Clot (1999) : «En fait, le genre peut se définir comme l'ensemble des activités engagées par une situation, convoquées par elle. II est une sédimentation et un prolongement des activités conjointes antérieures...ce qui a été fait auparavant par les générations d'un milieu donné, les manières dont les choix ont été tranchés jusque-là dans ce milieu, les vérifications auxquelles il a été procédé, les coutumes que cet ensemble rassemble » (p.37).

\section{Approches théoriques de la corporéité}

Les recherches qui problématisent l'agir humain du point de vue de la corporéité couvrent 
eJRIEPS 38 avril 2016

un champ théorique très large: l'ethnologie, l'anthropologie, l'histoire, la sociologie éclairent d'une manière spécifique l'agir social. Dans nos travaux, nous prenons appui sur trois courants de recherche, d'une part, l'ethnologie avec les travaux de Mauss (1950, 1947) sur les techniques du corps, d'autre part, l'approche phénoménologique qui considère "l'expression primordiale du corps » dans une situation d'intersubjectivité (Galimberti, 1998 ; Merleau-Ponty, 1945), enfin, l'anthropologie historique qui étudie la mimesis sociale (Gebauer \& Wulf, 2004 ; Joas, 1999 ; Wulf, 2007).

Avec les travaux de l'ethnologue Marcel Mauss, les techniques du corps représentent une approche descriptive des manières de faire des populations indigènes. Selon l'ethnologue, les «techniques du corps » montrent leur efficacité à travers la dimension corporelle de cet agir. Mauss souligne que "Le corps est le premier et le plus naturel instrument de l'homme ou plus exactement, sans parler d'instrument, le premier et le plus naturel objet technique, en même temps moyen technique de l'homme, c'est son corps.» (1950, p. 372). Lorsque Mauss décrit les actes traditionnels de la vie quotidienne, il considère la correspondance entre la gestuelle et le résultat de l'activité. Ainsi, les façons de porter, marcher, qui trouvent une explication en analysant le milieu de vie, sont étudiées du point de vue de leur potentiel de réalisation.

La corporéité sera également mise en évidence par le courant phénoménologique et, en particulier, par les travaux de Merleau-Ponty $(1945 ; 1962)$. Le philosophe conceptualise le corps comme "ouverture originaire au monde". Le sujet agissant appréhende ce qui l'environne non pas comme un simple fait objectivable mais comme un phénomène qui traverse son existence et qu'il investit subjectivement : "Tout usage humain du corps est déjà une expression primordiale »(Merleau-Ponty, 1962, p. 108). C'est dire que la corporéité du sujet constitue un rapport au monde qui se donne à voir et qui, par conséquent, peut être perçu par autrui. L'approche phénoménologique contribue à problématiser le corps comme un vecteur de relation sociale. Dans cette perspective la relation d'accompagnement entre un formateur et un professeur novice relèverait d'une intercorporéité. Autrement dit, la posture respective du formateur et celle du professeur stagiaire seraient perceptibles et interprétables aussi bien par les protagonistes que par un observateur extérieur. Un exemple de la lisibilité d'une posture est donné avec la description sartrienne du garçon de café. Sartre (1943) montre non seulement le jeu social révélé par la posture du garçon de café mais de surcroît met en évidence le jeu précis de celui qui «joue » à être garçon de café (Sartre a décrit finement les faits et gestes du garçon de café dans un but bien différent de celui qui nous occupe ici et qui renvoie à la 


\section{eJRIEPS 38 avril 2016}

mauvaise foi). L'étude de ce texte sous l'angle des gestes de métier, entendus comme gestes invariants d'une activité professionnelle, permet de considérer la puissance performative des gestes qui caractérisent l'agir professionnel du garçon de café.

"Considérons ce garçon de café. Il a le geste vif et appuyé, un peu trop précis, un peu trop rapide, il vient vers les consommateurs d'un pas un peu trop vif, il s'incline avec un peu trop d'empressement, sa voix, ses yeux expriment un intérêt un peu trop plein de sollicitude pour la commande du client, enfin le voilà qui revient, en essayant d'imiter dans sa démarche la rigueur inflexible d'on ne sait quel automate, tout en portant son plateau avec une témérité de funambule, en le mettant dans un équilibre perpétuellement instable et perpétuellement rompu, qu'il rétablit perpétuellement d'un mouvement léger du bras et de la main. Toute sa conduite nous semble un jeu... Mais à quoi joue-t-il ? ... il joue à être garçon de café...le garçon de café joue avec sa condition pour la réaliser ». (Sartre, 1943, p.95)

Cette description met en évidence l'investissement corporel de l'acteur dans son activité et montre la façon typique d'agir du garçon de café. Ses gestes de garçon de café sont perçus comme tels parce qu'ils appartiennent à un fond commun de pratiques sociales et qu'ils renvoient au genre de l'activité (Clot, 1999). Cette description met en évidence la relation étroite entre la posture du garçon de café « qui joue à être garçon de café » avec les gestes mobilisés pour donner vie à cette posture.

Un troisième courant de recherche, celui de l'anthropologie historique allemande (Gebauer \& Wulf, 2004) et la sociologie de l'agir (Joas, 1999) contribuent à la compréhension de la corporéité dans l'agir. Ces approches théoriques offrent des cadres qui accordent une grande importance au corps agissant, à la gestualité, au non verbal. L'idée majeure réside dans le fait que le corps unirait le sujet au monde dans sa capacité à intérioriser la société. Autrement dit, la plasticité du corps fait de celui-ci un médiateur entre le sujet agissant et le monde. Cette conception de la corporéité nous conduit à envisager le concept de posture tel qu'il a été élaboré par la psychosociologie pour caractériser l'agir professionnel d'un enseignant, d'un formateur. Si la posture est pour Ardoino (1990) un "système d'attitudes et de regards vis-à-vis des partenaires, des situations, des objets » dans une situation donnée, elle indique la manière dont l'acteur se positionne dans un contexte social donné, la façon dont la posture extériorise une intention d'agir qui se manifestera non seulement dans l'action auprès d'autrui mais aussi à travers des actes précis que sont les gestes professionnels.

Le courant de l'anthropologie historique marquera ses analyses par le fait que les gestes 


\section{eJRIEPS 38 avril 2016}

sont incorporés et qu'ils traduisent des filiations, des prolongements des gestes observés et mobilisés de façon quasi « naturelle ». Gebauer et Wulf (2004) conçoivent l'agir humain comme un remodelage incessant d'actes, de pratiques héritées, combinées, transformées. Le concept de mimesis sociale est un concept dynamique qui intègre la reprise de pratiques existantes et leurs modifications, d'où la possibilité d'une activité créative à partir d'un déjà-là. De ce point de vue, il est possible de concevoir le formateur comme un sujet social, historique, culturellement situé et dont la gestuelle possède un air de famille avec le genre de l'activité professionnelle.

Ces différents champs de recherche défendent l'idée que l'activité humaine dépend d'un investissement corporel, où la part du non verbal est reconnue comme complément de la communication linguistique. La posture et les gestes professionnels révèlent à notre sens la corporéité de l'agir. C'est avec ces éléments constitutifs de l'agir que le formateur entre en relation avec les formés. II nous reste maintenant à préciser les caractéristiques du geste professionnel.

\section{La reconnaissance des gestes professionnels}

L'agir professionnel du formateur peut être étudié sous l'angle des gestes de métier (Clot, 1999) en cherchant à identifier les gestes invariants de l'activité. II peut également être étudié dans une perspective différente, selon une conception de la professionnalité du formateur au sens donné par le sociologue Aballéa (1992) à la professionnalité comme la mobilisation d'une expertise et d'une déontologie. Ce qui signifie que les gestes professionnels auraient une dimension performative dans l'interaction entre formateur et formé : ils seraient non seulement adressés mais aussi marqués par leur efficacité. Dans nos travaux, nous avons cherché à spécifier les gestes professionnels (Jorro 2002, 2010, 2011, Pana-Martin 2015) d'un quadruple point de vue :

- Iorsqu'ils se déploient dans un espace intersubjectif et qu'ils sont adressés,

- lorsqu'ils sont mobilisés au bon moment et manifestent le sens du kaïros,

- Iorsqu'ils témoignent de l'engagement du professionnel et qu'ils expriment une amplitude,

- $\quad$ lorsqu'ils cherchent à transmettre un message.

Reprenons chaque trait distinctif du geste professionnel.

\section{- L'adresse du geste}




\section{eJRIEPS 38 avril 2016}

Le geste professionnel s'inscrit dans un espace intersubjectif où l'existence de l'autre est reconnue. L'interaction se transforme en interrelation. Avec l'adresse, on peut donc considérer le fait de se soucier de l'autre, car faire un geste c'est penser à l'autre. Cette forme expressive de l'agir peut être ressentie par les apprenants. L'exemple de Camus (1994) montre la portée éducative des gestes professionnels.

La dimension intersubjective de la relation formative suppose l'acceptation de la différence, et l'idée que l'on peut aller à la rencontre d'autrui. Deleuze (2008) parle d'une " double capture » dans la relation ; le formateur assumerait son devenir-formé, en faisant en sorte de favoriser les conditions de l'apprentissage, et le formé son devenir-formateur en gagnant une autonomie nouvelle dans l'étude. Recevoir un geste ou faire un geste, c'est tendre vers une rencontre interpersonnelle; le geste n'est pas seulement un signal avec lequel l'apprenant doit obtempérer, il est une invitation à comprendre et à agir.

Mais le geste professionnel est doublement adressé, d'une part vers celui qui le met en œuvre et d'autre part vers le destinataire. Autrement dit, le formateur agissant d'une certaine manière serait donc doublement conscient de l'effet de son agir.

\section{- Le sens du kaïros}

Agir avec le sens du kaïros, c'est agir avec le sens de l'improvisation. Le formateur saisit le moment opportun pour intervenir, fait preuve de prise de risque, joue sur le déroulement de l'action prévue pour lui donner une autre teneur. L'imprévu est transformé en situation favorable. Detienne et Vernant (1974) ont dénommé la mètis l'acte inventif qui prend appui sur l'occasion favorable. Le sens du kaïros apparaît dans le geste opportun. La créativité de l'acteur résulte de cet art de l'improvisation puisqu'il s'agit de jouer avec les temporalités, mais aussi de manœuvrer avec les cadres de la situation (Goffman, 1973). Art du jongleur, art de l'éventail, autant d'expressions métaphoriques pour valoriser les ajustements dans la gestualité du praticien.

\section{- L'amplitude du geste}

Dans le contexte d'une situation de formation, les gestes professionnels se déploient sur fond d'indétermination tant la situation d'apprentissage est dense. L'engagement du formateur peut être décisif pour la conduite de la formation. En effet, une situation est toujours traversée d'imprévus. Les gestes mobilisés peuvent avoir une amplitude différente de ceux qui avaient été envisagés parce que l'interaction en formation oppose une réalité autre. Cette capacité à ajuster une activité en situation, à la développer, suppose tout à la fois un sens de l'action et un engagement professionnel. Avec l'engagement, on saisira alors l'empan du geste professionnel, geste minimaliste ou geste 


\section{eJRIEPS 38 avril 2016}

de développement de la situation. Pana-Martin (2015) a identifié l'amplitude du geste comme une caractéristique déterminante de l'agir professionnel du formateur accompagnant des enseignants débutants.

\section{- Le message éthique du geste éducatif}

La dimension professionnelle du geste réside aussi dans sa mesure, sa retenue. De nombreux pédagogues ont insisté sur les effets nocifs de la relation autoritaire pour insister sur les valeurs de respect et de considération. Une dimension éthique traverse la gestualité. Par les gestes qu'il mobilise, le formateur peut installer les conditions d'une situation de formation propice aux apprentissages. Les gestes éthiques supposent des gestes de retenue dans le dialogue: le silence, la tolérance à l'égard des réflexions encore tâtonnantes sont des indicateurs d'une attitude d'écoute et de respect... Par ailleurs, le format de l'appréciation tend à mettre en évidence le positionnement de juge ou d'ami critique. La posture éthique du formateur peut ouvrir sur une relation d'accompagnement. Ainsi, nous avons identifié (Jorro, 2000) des gestes évaluatifs qui établissent une relation de suture ou d'autonomie. De tels gestes délivrent un message éducatif vers le formé ou le groupe de formation.

$\mathrm{Si}$ dans le contexte scolaire un certain nombre des gestes professionnels sont manifestement mobilisés pour agir sur la construction d'une micro-culture de classe, en formation il peut être concevable que les gestes du formateur autorisent des dynamiques individuelles et collectives de travail. Gebauer et Wulf (2004) insistent sur l'effet d'intégration sociale joué par les gestes. A la fonction de socialisation du geste, se greffe la dimension symbolique puisque le propre du geste humain est de signifier au-delà de sa simple existence de fait. La corporéité de l'activité opère dès qu'un groupe perçoit le corps hésitant du formateur, le corps instituant du formateur chevronné. Ces deux gestuelles, (entendues comme systèmes d'attitudes et de gestes formant une cohérence interne), qui mobilisent des dimensions non verbales et des pratiques langagières constituent des " expressions primordiales » interprétées par les adultes. Le corps parlant du formateur est donc le vecteur de processus de sémiotisation.

\section{Le dispositif de recherche}

La recherche conduite sur 18 mois a concerné 13 formateurs d'enseignants du nord de la France. A raison de séminaires d'un jour et demi tous les trois mois, la recherche collaborative a porté sur l'accompagnement d'enseignants débutants du $1^{\mathrm{er}}$ degré et, en particulier, sur la situation d'entretien post observation de séance de classe choisie par les 


\section{eJRIEPS 38 avril 2016}

formateurs en fonction de leurs préoccupations et de leur intérêt pour la discipline (mathématiques, français). Une dizaine de situations ont fait l'objet de séances d'analyses de pratique.

La construction du dispositif de recherche résulte d'un processus de négociation avec les acteurs, lesquels ne souhaitaient pas être filmés mais acceptaient de puiser dans leurs expériences professionnelles pour les partager en grand groupe et les soumettre à l'analyse collective. La vidéoformation a été utilisée sur des situations qui ne concernaient pas directement les formateurs et parce qu'elles permettaient une décentration par rapport aux situations qui étaient proposées à l'analyse du groupe. Les analyses de vidéos constituaient en quelque sorte un « pré travail d'analyse » des attitudes corporelles tenues en classe par les enseignants. Le rapport à l'image étant délicat pour certains membres du groupe la démarche collective d'analyse de pratiques a été décidée.

Les matériaux recueillis sont constitués de traces orales des formateurs (présentations de situations d'accompagnement, interprétations premières puis secondes des situations vécues et proposées à l'analyse du groupe), de synthèses de séances de travail et de traces écrites produites par le groupe entre les séminaires (réflexions personnelles, autoanalyses de quatre séances d'entretien enregistrées).

Deux grilles ont été produites afin d'une part, de permettre l'analyse des situations et d'autre part, de caractériser les postures et gestes professionnels.

La grille d'analyse des situations d'accompagnement prend en compte le contexte éducatif, la description de l'interaction, la relation établie entre le formateur et le professeur novice, la nature de l'interaction langagière et le contenu de l'échange. Cette première grille permet de contextualiser la situation d'accompagnement et livre des indications sur la place respective de chaque professionnel.

1 - Description de la situation d'accompagnement professionnel (contexte éducatif, classe, séquence observée, expérience de l'enseignant)

2 - Relation formateur-enseignant: places de chacun dans l'entretien (se faire face, être côte à côte et attitudes : coopérative, retrait, esquive...)

3 - Interaction langagière : écoute/ dialogue/ monologue démonstration/ évaluation, co-évaluation

4 - Contenu de l'échange : analyse du déroulement de la séquence : clarification didactique, gestion de la classe, rapport aux élèves, réflexivité de l'enseignant (auto-évaluation), régulation de l'activité.

La grille d'analyse de la posture et des gestes professionnels se focalise sur l'attitude physique et mentale du formateur et du professeur novice. Elle vise à identifier également la tonalité de l'interaction langagière avec les mouvements du corps, la présence d'une relation d'étayage avec la manifestation d'actes précis. 
- $\quad$ Attitude du formateur : positionnement physique et intention exprimée

- Attitude du professeur novice : positionnement physique et intention exprimée

- Attitude du formateur : prise de parole, regard, écoute, usage d'artefact...

- Attitude du professeur novice : prise de parole, regard, écoute, usage d'artefact...

L'analyse et l'interprétation des données recueillies suit trois étapes: description individuelle, puis recoupement des analyses en vue de l'élaboration d'une synthèse collective et travail d'inférence à partir de l'identification d'une attitude ou d'un acte spécifique.

L'analyse de la situation d'accompagnement par chaque participant a permis l'identification d'attitudes chez le formateur. La récurrence des analyses produites et leur recoupement a été déterminante pour inférer les postures des formateurs. La démarche d'identification des gestes professionnels a suivi le même processus : croisement des analyses et travail d'inférence pour interpréter les actes perçus comme décisifs dans l'agir du formateur.

A l'issue de ce travail d'analyse et d'interprétation, deux résultats de recherche sont identifiés :

- le premier consiste en la caractérisation de postures professionnelles qui favoriseraient l'accompagnement des acteurs.

- Le second résultat concerne l'identification de gestes professionnels reliés aux postures professionnelles identifiées.

\section{Des postures de formateur dans un processus d'accompagnement professionnel}

Le premier résultat de cette recherche réside dans l'identification d'intentions d'agir spécifiques de l'accompagnement professionnel d'enseignants débutants. Les formateurs conscientisent des intentions qui leurs «tiennent à cœur " et qu'ils convertissent en attitude auprès d'eux. Ils explicitent ainsi leur posture de formateur accompagnant des professionnels débutants. Nous avons précisé en introduction que la posture était définie par Ardoino (1990) comme un système interne d'attitudes dans une situation donnée (Mulin, 2014). Pour Charlier \& Biemar (2012), Paul (2004), la posture renverrait à une disposition psychique et corporelle.

Dans notre recherche, cinq postures ont été distinguées pour caractériser l'activité du formateur en situation d'entretien post observation de classe. Comme présenté précédemment ces postures sont inférées à partir des analyses de pratiques. Nous les présentons successivement de manière synthétique : 
eJRIEPS 38 avril 2016

- La posture de pisteur revient à organiser la situation d'entretien de sorte que l'enseignant trouve ses repères et comprenne les règles du jeu. Le terme métaphorique de pisteur signifie que le formateur balise la situation d'entretien de sorte que l'enseignant débutant puisse réfléchir dans de bonnes conditions à son activité professionnelle. Le formateur garantit le cadre de l'entretien et renvoie un message de réassurance à l'enseignant. Le fait d'organiser les places respectives de chacun et d'aménager l'espace de travail pour mener à bien l'entretien renvoie au souci d'installer une situation propice à l'échange.

\begin{abstract}
"Avant l'entretien je m'étais donné plusieurs points d'attention, je souhaitais que l'échange soit d'environ 45 minutes car il me semblait que ce temps était suffisant, du point de vue de l'effort de concentration, d'écoute. J'ai veillé à préciser dès le début que nous prendrions 40 à 45 minutes. J'ai donc annoncé à $\mathbf{X X X}$ dès le début qu'elle prendrait d'abord la parole et que l'on travaillerait à partir de ses questions et éventuellement je rajouterais quelques points. Je dis souvent "éventuellement " pour les rassurer et montrer que mon intervention n'est pas systématique. Je préfère partir de ce qu'elle dit pour commencer... » (Autoanalyse écrite)

" je préfèrerais, si tu le veux bien, que ce soit toi qui commence et qui choisisse les points à aborder: si çà se trouve ce seront les mêmes que ceux que j'ai relevé d'ailleurs. " (entretien avec professeur novice rapporté en situation d'analyse de pratiques)
\end{abstract}

- La posture de contradicteur traduit la manière dont le formateur entre dans une écoute active et constructive. En cherchant à entendre la parole de l'enseignant, le formateur s'intéresse aux non dits, aux significations cachées. Cette posture renvoie à l'art de la parezzia (Foucault, 2009) ou art du questionnement qui cherche à interpeller les évidences, les idées préconçues de l'enseignant. La posture de contradicteur suppose toutefois une lecture critique de la situation ainsi qu'une lecture bienveillante et exigeante.

- «Pendant l'entretien j'ai été concis dans mes interventions afin de permettre à mon interlocutrice de bénéficier d'un maximum de temps pour s'exprimer, réfléchir. En fait, je voulais réagir après avoir entendu ce qu'elle avait envie de dire et ça... c'était vraiment bien... elle avait plein de choses à dire. Je la laissais parler...pour revenir sur un point qui me paraissait pas clair dans sa pratique... j'ai pris le temps de lui dire "êtes-vous sûre que votre intention de départ a été comprise par la classe ? Je savais que j'allais la perturber...oui je trouvais intéressant de lui donner une autre lecture de sa séquence. En fait, elle a été silencieuse puis m'a posé des questions ...c'est moi qui était sur la sellette (rire !) ...on a commencé par reprendre la situation dans l'objectif qu'elle voulait atteindre et on a comparé avec l'activité des élèves...avec la tâche d'écriture, comment elle avait demandé aux élèves de la réaliser... donc... c'était constructif pour elle car elle voyait bien qu'il manquait quelque chose alors on a cherché ensemble ce qui pouvait décoincer le groupe d'élèves qui semblait... ». (projet d'écriture en classe de CM1) (autoanalyse orale)

- La posture de traducteur est une posture importante car elle explicite auprès de l'enseignant des notions ou des aspects qui échappent à la compréhension du professionnel. Le traducteur rend accessible des points non problématisés, il peut 
eJRIEPS 38 avril 2016

débusquer des impensés ou encore identifier des représentations erronées à propos d'un champ disciplinaire, d'une notion à enseigner.

- "j'ai souligné que la notion introduite (plus grand que) était plus importante que la présentation qu'elle avait faite et que sa séquence devait évoluer vers une séance où la comparaison devait se faire avec d'autres objets que les gommettes (de différentes tailles) sinon les élèves ne pourraient pas réutiliser la comparaison. On a cherché ensemble des situations qui pourraient être investies avec les objets présents dans la classe et dans l'école pour ce type d'approche ".(Autoanalyse écrite d'une séquence de mathématique en maternelle, notions plus grand que, plus petit que). "

Son activité consiste à engager le praticien à approfondir une réflexion sur un aspect particulier de son activité professionnelle, à faire en sorte que l'enseignant exerce une vigilance plus forte sur des aspects non vus.

La posture de médiateur est une posture marquée par la volonté de faire lien : lien avec des ressources didactiques, liens avec des personnes ressources qui pourraient être utiles pour l'activité de l'enseignant. Le formateur peut concevoir ses propres limites et renvoyer le praticien vers d'autres étayages. C'est aussi une posture qui permet de soutenir les efforts accomplis par le praticien et de valoriser l'engagement de l'enseignant. C'est également une posture qui mobilise des gestes de conseil.

- «A ce moment là, j'ai hésité pour trouver une réponse, je me suis retrouvé à court sur ce type d'écrit car il me manque des notions didactiques ou autres... c'est un peu gênant mais je préfère le dire...et j'hésite pas à dire ...plutôt que de rester évasif ... je préfère dire que je vais voir et qu'on reviendra dessus que je vais me renseigner même ça peut m'arriver de dire au professeur d'en parler avec un autre formateur... " (autoanalyse orale)

Situation qui arrive dans le premier degré où la polyvalence est affichée et suppose de nombreuses connaissances didactiques que les formateurs peuvent ne pas maîtriser.

- La posture de régulateur : le formateur tente de mettre en situation d'autoévaluation ou de co-évaluation l'enseignant afin qu'il repère ses points d'appui et entrevoit également des pistes d'action. Des gestes de valorisation et d'ajustement sont mobilisés par le formateur dans cette posture. Parfois, l'approche déficitaire de l'enseignant sur sa propre pratique est perçue par le formateur qui cherchera à valoriser les réussites de l'activité tout autant qu'à rassurer sur les enjeux de développement professionnel.

- " presqu'à la fin de l'entretien, je demande aux professeurs comment ils voient leur activité, en quoi ils se sentent à l'aise... où ils ont le sentiment d'avoir à travailler. Je les incite à porter un regard sur ce qu'ils savent faire. Certains professeurs débutants ont tendance à dire qu'ils savent pas trop...mais en fait, ils me disent là où ils se sentent en insécurité...ils ont une vision parfois un peu sévère.. mon rôle consiste à les rassurer...j'essaie de les aider à voir des possibilités d'amélioration...c'est nécessaire... c'est intéressant de les encourager dans ce sens et de leur montrer que l'expérience ça sert aussi...qu'ils 
eJRIEPS 38 avril 2016

commencent juste et que ça va venir..."(autoanalyse orale)

\section{Le geste professionnel comme manifestation d'une posture professionnelle}

Le second résultat de notre recherche consiste en la relation étroite entre la posture et les gestes professionnels. Ainsi, le geste professionnel dépend d'une intention professionnelle actualisée par la médiation d'une posture. Nous présenterons ici des gestes professionnels articulés à deux postures qui sont dominantes dans l'activité du formateur. Les gestes professionnels dans la posture de traducteur :

- Le geste de désignation : Le geste de désignation renvoie à un geste récurent du champ de l'éducation et de la formation. II peut porter sur un objet, une situation, une action. Ce geste entraine une clarification auprès du destinataire. Le fait de montrer une référence, de nommer un point critique «on va s'arrêter sur ce point... » permet de lancer la réflexion sur une dimension précise. Le formateur montre ainsi la vigilance exercée sur des détails qui peuvent entrainer des difficultés en classe.

- Le geste explicatif : par ce geste, le formateur peut revenir sur une notion, la rendre accessible au formé, la situer dans un contexte pour permettre sa compréhension : "tu vois quand tu abordes cette notion eh bien d'abord c'est compliqué pour les élèves mais plutôt que d'entrer dans une explication tu pourrais d'abord leur demander comment eux ils comprennent le sens de cette phrase...ton explication elle vient trop tôt... » Le geste explicatif permet de lever les malentendus, les contresens lorsqu'il s'agit de travailler sur un champ disciplinaire, sur une démarche, sur l'installation d'une séquence et la mise en activité des élèves.

- Le geste d'institutionnalisation : le formateur peut soutenir la démarche entreprise par l'enseignant en soulignant l'intérêt de la séquence observée, la pertinence de l'action du praticien, en validant le contenu de la séquence. Ce geste est très attendu des enseignants débutants, il a un effet rassurant parce qu'il témoigne d'une forme de reconnaissance du travail conduit et donc pensé, élaboré construit avec la classe. Le geste d'institutionnalisation est un geste qui fait autorité parce qu'il porte un message de validation.

"Et là je reviens sur ce qu'elle a réussi, ça fait sens pour elle et moi je veux lui montrer que c'est important.. c'est pour ça que j'insiste ...ce qu'elle a obtenu de l'élève c'est vraiment une possibilité d'apprendre à écrire et le projet, il peut aussi conduire à ce type de comportement...à un moment il y a une envie de faire... je lui dit que c'est une réussite pour cet élève d'avoir écrit ce petit texte pour sa carte postale... " (autoanalyse orale d'une situation d'écriture de carte postale au CE2-CM1) 
Les gestes professionnels dans la posture de contradicteur :

- Le geste de questionnement : le formateur invite le praticien à prendre la parole et à faire part de sa réflexion sur l'activité vécue. Il incite à l'analyse et au dégagement de points qui importent à l'enseignant. Le geste de questionnement tend à montrer l'importance que le formateur accorde à la réflexion de l'enseignant et la prudence qui est la sienne pour permettre une relation dialogique dont l'effet sera bénéfique pour le praticien.

"je lui demande si elle peut me dire ce qu'elle pense de l'attitude de l'élève quand il répond aussi vite et qu'il répond sans entendre la fin de la question, ce qu'elle peut faire pour le freiner pour éviter qu'il apporte une idée à côté. Je la pousse à envisager un scénario comme si elle pouvait remonter le temps pour comprendre ce qui pourrait être mis en place à ce moment là. En fait, au début c'est le grand silence, elle cherche et moi j'attends de lui retourner la question qui me paraît importante: est-ce que c'est une question de discipline ou une manière de présenter le savoir ?" (autoanalyse écrite)

- Le geste de problématisation: le formateur considère comme nécessaire de sensibiliser l'enseignant sur les décalages perçus dans le feu de l'action et ce que l'observateur a lui même pu observer. A partir des comportements d'élèves, de traces écrites, de consignes données, ces décalages font l'objet d'un échange entre les deux professionnels et le geste de problématisation revient à émettre des hypothèses sur le déroulement de l'activité. En suscitant le point de vue du praticien sur les décalages, le formateur cherche à faire problématiser l'activité par l'enseignant.

« J'avais vraiment envie pour cette situation de correction de grammaire... qu'elle essaie de réfléchir là-dessus, la correction de l'exercice...ça tourne un peu au jeu des questionsréponses sans aller plus loin. Comment elle pourrait faire pour que ce soit un temps de recherche collectif et de réflexion sur des hypothèses. Je voulais qu'elle interroge un peu ce que font les élèves pendant le temps de correction : un concours de la bonne réponse ? un travail de recherche ? Comment s'y prendre pour que la correction ne devienne pas une course à la bonne réponse? Comment elle pouvait prendre un peu de recul pour interroger l'efficacité de ce temps de correction pour les élèves ? Le paradoxe...corriger oui mais pour qui ?(autoanalyse écrite, Classe de CM1)

Les gestes de questionnement et de problématisation tendent à montrer l'attitude d'enquêteur tenue par le formateur. Ce dernier n'est pas seulement spectateur d'une situation d'enseignement, ni récepteur de l'interprétation donnée par le praticien. II contribue par sa position externe à l'approfondissement de la réflexion. La posture de contradicteur est donc une posture de recherche qui mobilise une gestuelle particulière : 


\section{eJRIEPS 38 avril 2016}

intensité du regard, précision langagière dans le questionnement, attitude interrogative plutôt qu'assertive, sollicitation non verbale (regard, sourire) du point de vue du praticien.

\section{Eléments de discussion}

Trois éléments de discussion seront ici présentés. Tout d'abord, une réflexion sur la démarche qualitative qui a été retenue. La recherche prend en compte une parole qui reconstruit une expérience d'entretien post observation de classe. Le matériau est donc constitué d'un discours qui intègre des séquences narratives, explicatives et argumentatives que le professionnel livre et que la recherche considère comme légitime. Le travail de recherche consiste donc, dans un premier temps, à élaborer une analyse sur un matériau langagier imprégné d'un vécu et non pas sur un matériau enregistré en audio ou encore sur une trace vidéographique d'une situation donnée. Le fait de se doter de deux grilles d'analyse permet de croiser les indices prélevés dans le discours tenu par le formateur qui expose une expérience d'entretien. La première grille insiste sur le contexte de l'interaction, la seconde est centrée sur la relation et par conséquent sur la spécificité de l'intercorporéité. Ces deux grilles permettent de partager un cadre d'analyse et, chemin faisant, de caractériser les intentions sous-jacentes du formateur dans l'accompagnement du professeur novice. Les auto-analyses orales en séance d'analyse des pratiques et les 4 autoanalyses écrites rédigées livrent des indices qui nous paraissent cohérents et qui nous ont permis de caractériser des postures et des gestes professionnels.

Le second point de discussion concerne la caractérisation des postures dans la situation d'accompagnement professionnel. Nous avons souligné comment les approches théoriques issues de la phénoménologie et de l'anthropologie historique rendaient compte du pouvoir médiateur de la posture dans l'espace social. Cette recherche met en évidence des intentions précises chez les formateurs qui s'expriment à travers des attitudes avec les gestes qui en découlent. Ces postures ne sont pas mobilisées par tous les formateurs lors de chaque entretien, elles apparaissent parfois par défaut quand le style du formateur fait apparaître une posture dominante. Par exemple, la posture de traducteur apparaît plus souvent que celle de régulateur. II paraît plus aisé aux formateurs de revenir sur un point de la séance, d'apporter des explications au besoin afin de consolider un savoir requis dans la séance par l'enseignant. La posture de régulateur est peu identifiée car les formateurs sont réticents par rapport à l'évaluation de l'activité professionnelle. Ils se défendent de cette posture; ce que la recherche a déjà montré (Jorro, 2110). Les postures d'accueil et d'écoute ont été étudiées au cours de plusieurs séances pour 


\section{eJRIEPS 38 avril 2016}

pouvoir être distinguées. La posture de médiation est apparue au fur et à mesure des analyses. La palette des postures ainsi mise au jour permet d'envisager une approche modale de l'agir du formateur : il serait possible en formation de sensibiliser les acteurs aux intentions du formateur dans l'interaction afin de favoriser l'élargissement et à la complexification des manières d'agir.

Le troisième point de discussion est relatif à l'identification de gestes professionnels qui prolongent et extériorisent la posture du formateur. Les gestes professionnels sont étroitement reliés aux postures dès lors qu'ils traduisent concrètement une intention. En tant qu'actes effectués par le formateur, ils indiquent l'intention du formateur. Nous avons évoqué le double adressage des gestes dans la mesure où le formateur agit en fonction de ce qu'il perçoit de la situation et dans le même temps agit envers le professeur. II destine son geste à autrui tout autant qu'à lui-même. Mais il serait plus juste de dire qu'il y a un triple adressage dès lorsque le geste est mis en œuvre dans un contexte institutionnel et organisationnel spécifique. Les matériaux ne nous ont pas permis de rendre compte précisément de l'influence du contexte dans la mobilisation d'un geste. De même, il aurait été intéressant de croiser les analyses des formateurs avec ceux des professeurs vers lesquels ces gestes étaient adressés. Si l'on considère la relation d'entretien comme une action conjointe, ces données deviennent nécessaires. Dans une recherche antérieure conduite auprès de conseillers pédagogiques du premier degré, les effets de ces gestes auprès des professeurs novices ont été identifiés (Jorro et PanaMartin, 2012). Lorsque le geste est adressé et après que la situation a été vécue comme constructive, les professeurs évoquent les effets de reconnaissance professionnelle de certains gestes adressés par les formateurs.

\section{Conclusion}

Cette recherche exploratoire nous incite à considérer ces premiers résultats avec prudence. La généralisation autour des postures et des gestes professionnels suppose d'autres travaux en ce sens. Une question reste en suspens. Peut-on identifier des postures similaires dans l'accompagnement des enseignants ayant du métier? Par ailleurs, dans un contexte où le genre professionnel du formateur n'est pas institué, la recherche collaborative contribue à la caractérisation de postures d'accompagnement professionnel qui font sens pour les participants de la recherche. La posture professionnelle et les gestes professionnels représentent des savoirs de l'action pour le formateur. A la suite de Demailly (2008), il est possible de souligner que la rationalisation 


\section{eJRIEPS 38 avril 2016}

du travail relationnel suppose une clarification des positionnements des acteurs. Le travail relationnel qui a été présenté dans cet article du point de vue de la corporéité constitue un travail « intime » autrefois laissé à la liberté de l'acteur. L'interaction avec autrui interprété comme une intercorporéité nous semble une occasion favorable de développement professionnel pour les formateurs.

\section{Bibliographie}

Aballéa, F. (1992). Sur la notion de professionnalité. Recherche sociale, 124, 39-49.

Alin, C. (2010). La geste formation. Gestes professionnels et analyse des pratiques. Paris : L'Harmattan.

Ardoino, J. (1990). Les postures ou impostures respectives du chercheur de l'expert et du consultant. Les nouvelles formes de la recherche en éducation (pp. 22-34). Paris : Matrice Andsha.

Brière-Guenoun, F. (2015). Les gestes du professeur d'éducation physique en milieu difficile : de multiples compromis au service de la co-construction des savoirs. Carrefours de l'éducation, 40, 85-103.

Bucheton, D. (2009). L'agir enseignant : des gestes professionnels ajustés. Toulouse: Octares.

Charlier, E. \& Biemar, S. (2012). Accompagner : un agir professionnel. Bruxelles : De Boeck.

Clot, Y. (1999). La fonction psychologique du travail. Paris : PUF.

Croce-Spinelli, H. (2014). Gestes professionnels. In A. Jorro (ed), Dictionnaire des concepts de la professionnalisation (pp. 145-148). Bruxelles : De Boeck.

Deleuze, G. \& Parnet, C. (2008). Dialogues. Paris : Champs.

Demailly, L. (2008). Politiques de la relation. Lille : Septentrion.

Foucault, M. (2009). Le courage de la vérité. Gallimard. Paris : Gallimard.

Gebauer, G. \& Wolf, C. (2004). Jeux, rituels, gestes. Paris : Anthropos.

Galimberti, U. (1998). Les raisons du corps. Paris : Grasset.

Joas, H. (1999). La créativité de l'agir. Paris : E. du Cerf.

Hassan, R. (2010). Écrire au tableau entre pratique langagière et geste professionnel. Travail et Formation en Education, 5. [En ligne], 5 | 2010, mis en ligne le 04 juin 2010, consulté le 10 mars 2016. URL : http://tfe.revues.org/1053

Jorro, A. (2011). Comprendre l'agir professionnel en éducation et en formation avec 


\section{eJRIEPS 38 avril 2016}

Ricœur in A. Kerlan \& D. Simard (eds), Paul Ricœur et l'action éducative. Laval : PUL.

Jorro, A. \& Mercier-Brunel, Y. (2011). Les gestes évaluatifs de l'enseignant dans une tâche de correction collective. Mesure et évaluation en éducation, 34 (3), 27-50.

Jorro, A. (2010). Le développement professionnel des acteurs : une nouvelle fonction de l'évaluation ? In L. Paquay, C. Vannieuwehoven, P. Wouters (eds), L'évaluation, levier du développement professionnel ? (pp. 253-264). Paris : De Boeck.

Jorro, A. \& Croce - Spinelli, H. (2010). Le développement de gestes professionnels en classe de français. Le cas de situations de lecture interprétative. Pratiques, 145$146,125-140$.

Jorro, A. (2004). Le corps parlant de l'enseignant. Entente, malentendus, négociations. In Actes du colloque de l'AIRDF. Québec.

Jorro, A. (2004). Les formateurs et l'accompagnement des professeurs stagiaires dans l'écriture du mémoire professionnel ». In J-P. Benoit \& A. Gonin-Bollo (eds), Dix ans de mémoires professionnels (pp. 197-207). Paris : INRP.

Jorro, A. (2002). Professionnaliser le métier d'enseignant. Paris : ESF.

Jorro, A. (1998). L'inscription des gestes professionnels dans l'action. Questions vives, 18, 7-13.

Loquet, M. (2006). Analyse des gestes professionnels: illustration de «l'œil du maquignon » chez une formatrice en expression corporelle. Revue française de pédagogie, 157, 119-130.

Mauss, M. (1950). Anthropologie et Sociologie. Paris : PUF.

Mauss, M. (1947). Manuel d'ethnographie. Paris : Petite bibliothèque Payot.

Mercier-Brunel, Y. \& Jorro, A. (2009). La parole évaluative de l'enseignant. Les dossiers des sciences de l'éducation, 22, 9-24.

Merleau-Ponty, M. (1945). Phénoménologie de la perception. Paris : Gallimard.

Merleau-Ponty, M. (1960). Signes. Paris: Gallimard.Mulin, T. (2014). Posture professionnelle. In A. Jorro (ed), Dictionnaire des concepts de la professionnalisation (pp. 213-216). Bruxelles : De Boeck.

Pana-Martin, F. (2015). Les gestes professionnels des formateurs d'enseignants en situation d'accompagnement individualisé. Thèse nouveau régime. Cnam, Paris.

Paul, M. (2004). L'accompagnement: une posture professionnelle spécifique. Paris: l'Harmattan.

Pujade-Renaud, C. (1981). Le corps de l'enseignant dans la classe. Paris : ESF. 
eJRIEPS 38 avril 2016

Sartre, J-P. (1943). L'être et le néant. Paris : Gallimard.

Sensevy, G. (2001) «Théorie de l'action et action du professeur » in Baudoin, J-M. \& Friedrich, J. (eds) Théories de l'action et éducation (pp. 203-224). Bruxelles : De Boeck.

Wulf, C. (2007). Une anthropologie historique et culturelle. Paris : Téraèdre. 